\title{
A NOTE ON MODULAR GROUPS ${ }^{1}$
}

\section{MORRIS NEWMAN}

Let $\Gamma$ be the $2 \times 2$ modular group; that is, the group of $2 \times 2$ rational integral matrices of determinant 1 in which a matrix and its negative are identified. In a recent article [1] J. L. Brenner introduced the normal subgroup $\Delta(m)$ of $\Gamma$, defined as the smallest normal subgroup of $\Gamma$ containing

$$
\left(\begin{array}{ll}
1 & m \\
0 & 1
\end{array}\right) .
$$

Let $\Gamma(m)$ be the principal congruence subgroup of level $m$ of $\Gamma$; that is, $\Gamma(m)$ consists of all elements $A$ of $\Gamma$ satisfying

$$
A \equiv \pm\left(\begin{array}{ll}
1 & 0 \\
0 & 1
\end{array}\right)(\bmod m)
$$

Then Brenner proved in [1] that

$$
\Gamma(m)=\Delta(m), \quad 1 \leqq m \leqq 5
$$

and posed the question of determining whether or not $\Delta(m)$ contains a principal congruence subgroup for larger $m$. This question was settled in the negative for $m$ not a prime power by I. Reiner in his paper [4], and in [3] M. I. Knopp proved that the groups $\Delta(m)$ are of infinite index in $\Gamma$ for $m \geqq 6$ and so cannot possibly contain a principal congruence subgroup, these being of finite index always.

Knopp also points out that the question had been considered and settled by F. Klein and R. Fricke in their treatise on the modular functions.

In this note we wish to point out that $\Delta(6)$ is an interesting group.

In fact we prove

Theorem. The group $\Delta(6)$ is just $\Gamma^{\prime \prime}$, the second commutator subgroup of $\Gamma$.

Since the first commutator subgroup $\Gamma^{\prime}$ is a free group of finite rank $>1$ it follows that $\Delta(6)=\Gamma^{\prime \prime}$ is a countably infinitely generated free group and so of infinite index in $\Gamma$.

To prove the theorem we first remark

Lemma 1. Let $G$ be a group generated by two elements $\alpha, \beta$. Let $N$ be

Received by the editors December 11, 1961.

1 The preparation of this paper was supported by the Office of Naval Research. 
a normal subgroup of $G$ containing $[\alpha, \beta]=\alpha \beta \alpha^{-1} \beta^{-1}$. Then $N$ contains $G^{\prime}$, the commutator subgroup of $G$.

Proof. $G$ is abelian modulo $N$ and the conclusion follows.

We also have

LEMMA 2. $\Gamma^{\prime}$ is a free group of rank 2 generated by

$$
\alpha=\left(\begin{array}{ll}
2 & 1 \\
1 & 1
\end{array}\right), \quad \beta=\left(\begin{array}{rr}
2 & -1 \\
-1 & 1
\end{array}\right) .
$$

The commutator $[\alpha, \beta]$ satisfies

$$
[\alpha, \beta]=\left(\begin{array}{rr}
-1 & -6 \\
0 & -1
\end{array}\right)=\left(\begin{array}{ll}
1 & 6 \\
0 & 1
\end{array}\right) \text {. }
$$

Proof. The first part of the lemma is well-known (see [2]), the generators usually being taken as

$$
\left(\begin{array}{ll}
2 & 1 \\
1 & 1
\end{array}\right), \quad\left(\begin{array}{ll}
1 & 1 \\
1 & 2
\end{array}\right) .
$$

The second part of the lemma is a straightforward computation.

If we apply Lemma 1 with $G=\Gamma^{\prime}, N=\Delta(6)$ we conclude that

$$
\Delta(6) \supset \Gamma^{\prime \prime} \text {. }
$$

But $\Delta(6)$ is the smallest normal subgroup of $\Gamma$ containing

$$
\left(\begin{array}{ll}
1 & 6 \\
0 & 1
\end{array}\right) \text { and } \Gamma^{\prime \prime} \text { contains }\left(\begin{array}{ll}
1 & 6 \\
0 & 1
\end{array}\right) \text {. }
$$

Hence $\Gamma^{\prime \prime} \supset \Delta(6)$. Combining the inclusion relations we have $\Gamma^{\prime \prime}=\Delta(6)$ which concludes the proof of the theorem.

\section{REFERENCES}

1. J. L. Brenner, The linear homogeneous group. III, Ann. of Math. (2) 71 (1960), 210-223.

2. H. Frasch, Die Erzeugenden der Hauptkongruenzgruppen für. Primzahlstufen, Math. Ann. 108 (1933), 229-252.

3. M. I. Knopp, A note on subgroups of the modular group, Proc. Amer. Math. Soc. (to appear).

4. I. Reiner, Subgroups of the unimodular group, Proc. Amer. Math. Soc. 12 (1961), 173-174.

National Bureau of Standards, Washington, D. C. 